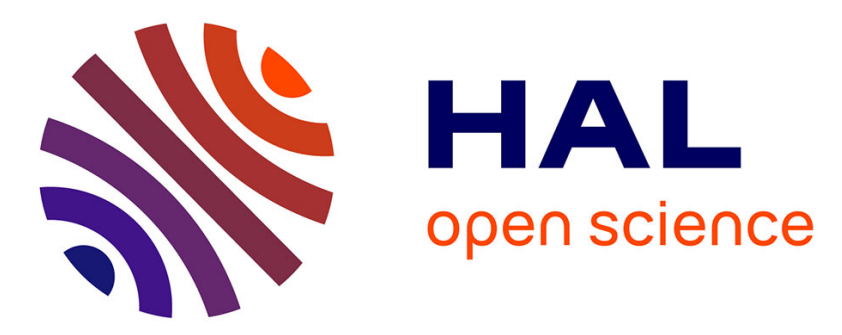

\title{
Users facing volume-based and flat-rate-based charging schemes at the same time
}

Patrick Maillé, Bruno Tuffin

\section{To cite this version:}

Patrick Maillé, Bruno Tuffin. Users facing volume-based and flat-rate-based charging schemes at the same time. 8th Latin American Network Operations and Management Symposium (LANOMS 2015), Oct 2015, Joao Pessoa, Brazil. pp.23-26. hal-01113322

\section{HAL Id: hal-01113322 \\ https://hal.inria.fr/hal-01113322}

Submitted on 5 Feb 2015

HAL is a multi-disciplinary open access archive for the deposit and dissemination of scientific research documents, whether they are published or not. The documents may come from teaching and research institutions in France or abroad, or from public or private research centers.
L'archive ouverte pluridisciplinaire HAL, est destinée au dépôt et à la diffusion de documents scientifiques de niveau recherche, publiés ou non, émanant des établissements d'enseignement et de recherche français ou étrangers, des laboratoires publics ou privés. 


\section{Users facing volume-based and flat-rate-based charging schemes at the same time}

\author{
Patrick Maillé \\ Telecom Bretagne \\ 2, rue de la Châtaigneraie \\ 35576 Cesson-Sévigné Cedex, France \\ Email: patrick.maille@telecom-bretagne.eu
}

\author{
Bruno Tuffin \\ Inria Rennes \\ Campus Universitaire de Beaulieu \\ 35042 Rennes Cedex, France \\ Email: bruno.tuffin@inria.fr
}

\begin{abstract}
In the Internet, the data charging scheme has usually been flat rate. But more recently, especially for mobile data traffic, we have seen more diversity in the pricing offers, such as volume-based ones or cap-based ones. We propose in this paper to study the behavior of heterogeneous users facing two offers: a volume-based one and a flat-rate one. On top of that selection, we investigate 1) the relevance for an ISP to propose the two types of offers, and optimize the corresponding prices, and 2) the existence of a solution to the pricing game when the offers come from competing providers.
\end{abstract}

\section{INTRODUCTION}

The common pricing scheme in the Internet is the socalled flat rate pricing, where a user pays a fixed subscription fee, and can use the network as much as he wants. But there is currently a trend in telecommunications to move to usage-based pricing schemes, where the price you pay depends on your consumption pattern. The reasons invoked for those moves generally involve arguments of congestion and fairness between users. That trend has been observed with the introduction of cap-based pricing in broadband access, and the definition of the charging schemes for wireless networks. For an extensive description of flat-rate versus usage-based pricing, see [1], as well as [2] for a general presentation and discussion about Internet economics.

With the appearance of new wireless technologies (and also the deployment of fiber-to-the-home solutions), users are faced with several options, that are not all priced the same way. In this paper we investigate the co-existence of solutions based on flat-rate pricing with others based on usage-based pricing. Users being heterogeneous in their preferences, their preferred scheme may differ. Focusing on the case of two alternatives (one flat-rate and one usage-based), we analyze the impact of this diversity of users and choices in two settings: in the first one the two solutions are proposed by a monopolist provider trying to maximize revenues, while the second one considers competing providers who set their pricing parameters to attract users in order to make revenue (hence a noncooperative pricing game). Discussions on this can be found in [2] and the references therein, see also [3], [4].

Our model is inspired by [5], where the choice of which scheme to prefer is studied, but not the coexistence of both schemes. The authors of [5] study the case of two schemes jointly proposed to users in [6] to analyze conditions for both schemes to be selected by users, but no revenue-maximization or competition problematics are considered. Our contributions can be summarized as follows:

- for a user with quadratic willingness-to-pay (in terms of the consumed quantity), we express analytically the choice made depending on the user-specific parameters and the parameters of both pricing solutions;

- $\quad$ then, assuming some distribution over user-specific parameters we numerically treat some examples of pricing decisions in the monopoly (revenue-maximization) and competition (non-cooperative game) cases.

- Our results suggest that, with our set of chosen parameters, offering the two options in the case of a monopoly increases very slightly the provider's revenue and has a limited impact on total demand. Also, when the options are offered by competitive providers, the pricing game will end up with a price war, i.e. null revenues, or very limited revenues if we introduce some data management costs in the model.

\section{MODEL: PRICING SCHEMES AND USER PREFERENCES}

We consider two ISPs (possibly controlled by the same entity) offering connectivity services to users. ISP 1 is implementing flat-rate pricing with access price $p_{1}$ per user (say, per month, like other values later on), after what the user can consume the amount of volume she wishes at no extra charge. ISP 2 on the other hand implements a volume-based pricing scheme, with still an access charge per user (and per month) $p_{2}$, but also a charge per unit of volume $q_{2}$, so that the (per month) total charge for a consumed volume $v$ is $p_{2}+q_{2} v$.

Users are assumed heterogeneous. We index them by a (one-dimensional) type parameter $\theta$ characterizing their valuation (willingness-to-pay) function. We consider without loss of generality a total user mass of 1 , and assume a density $f(\theta)$ for value $\theta$ with corresponding cdf $F$. Denote by $V(\theta, v)$ the amount (in monetary units) that a type- $\theta$ user is willing to pay for consuming a volume $v$ per month: $V(\theta, v)$ is assumed nondecreasing in $v$, and constant after some value $v_{\max }(\theta)$ that a type $\theta$ user is interested in getting (or that the provider can serve per user; in this case it may not depend on $\theta$ ) if the service were totally free.

We assume here that the networks are over-provisioned so that there is no QoS issue. This assumption is especially relevant for wired DSL or fiber access networks for instance. 


\section{USER BEHAVIOR}

We consider in this section that price parameters $p_{1}, p_{2}$ and $q_{2}$ are fixed, and study the choice for a particular user, together with her corresponding consumption level.

A user of type $\theta$, if associated to ISP 1 (the ISP implementing flat-rate pricing), will consume a volume $v=v_{1}^{*}(\theta)$ such that her net utility $V(\theta, v)-p_{1}$ is maximized, i.e.,

$$
v_{1}^{*}(\theta)=\operatorname{argmax}_{v \geq 0}\left(V(\theta, v)-p_{1}\right) .
$$

But since $V(\theta, \cdot)$ is increasing, we get $v_{1}^{*}(\theta)=v_{\max }(\theta)$.

Similarly, if associated to ISP 2 (that implements usagebased pricing), she will consume $v_{2}^{*}(\theta)$ with

$$
v_{2}^{*}(\theta)=\operatorname{argmax}_{v \geq 0}\left(V(\theta, v)-\left(p_{2}+q_{2} v\right)\right),
$$

(assuming that this argmax exists).

Overall, a type- $\theta$ user will select the option (among \{ISP 1, ISP 2, no ISP $\}$ ) maximizing her utility, considering that the "no ISP" choice yields a null utility. In case of equality between two options (marginal users), we assume that the user chooses the option yielding the largest $V(\theta, \cdot)$, maximizing the generated valuation (like a company trying then to maximize its turnover).

In practice of course, $p_{2}<p_{1}$ otherwise ISP 1 is always preferred to ISP 2. Assume as in our reference paper [5] that valuation functions are quadratic, of the form

$$
V(\theta, v)= \begin{cases}\theta v-\frac{v^{2}}{2 a} & 0 \leq v \leq \theta a \\ \frac{a}{2} \theta^{2} & v \geq a \theta\end{cases}
$$

with $a$ a constant and $\theta$ the (non-negative) user-specific parameter (her type). Such a valuation function corresponds to a demand $D(p, \theta)=a(\theta-p)^{+}$with $x^{+}:=\max (0, x)$ (for more, see [5]), and $v_{\max }(\theta)=a \theta$.

In that case $v_{2}^{*}(\theta)$ is obtained from the maximization of $V(\theta, v)-p_{2}-q_{2} v$, hence from the relation $\frac{\partial V}{\partial v}\left(\theta, v_{2}^{*}(\theta)\right)-q_{2}=$ 0 , leading to $v_{2}^{*}(\theta)=a\left(\theta-q_{2}\right)^{+}$. In words, $v_{2}^{*}(\theta)$ is the level of demand at unit price $q_{2}$.

The corresponding utilities for both choices are thus

$$
\begin{aligned}
V\left(\theta, v_{\max }(\theta)\right)-p_{1} & =\frac{a \theta^{2}}{2}-p_{1} \\
V\left(\theta, v_{2}^{*}(\theta)\right)-p_{2}-q_{2} v_{2}^{*}(\theta) & =\frac{a}{2}\left(\theta-q_{2}\right)^{2}-p_{2} .
\end{aligned}
$$

Summarizing, a type- $\theta$ user will choose her service option according to the following threshold rule.

$\begin{aligned} & \text { Proposition 1. Let } \theta^{-}:=\min \left(\sqrt{\frac{2 p_{1}}{a}},\right.\left.q_{2}+\sqrt{\frac{2 p_{2}}{a}}\right) \\ & \text { and } \theta^{+}:=\max \left(\sqrt{\frac{2 p_{1}}{a}}, \frac{q_{2}}{2}+\frac{p_{1}-p_{2}}{a q_{2}}\right) .\end{aligned}$

Then a type- $\theta$ user will

i) prefer not to join any provider if $\theta \in\left[0, \theta^{-}\right)$;

ii) choose the volume-based provider if $\theta \in\left[\theta^{-}, \theta^{+}\right)$;

iii) choose the flat-rate provider if $\theta \in\left[\theta^{+}, \infty\right)$.

In particular, if prices are such that $\sqrt{\frac{2 p_{1}}{a}} \leq q_{2}+\sqrt{\frac{2 p_{2}}{a}}$ then $\theta^{+}=\theta^{-}$and no user selects the volume-based provider.
Proof: The no-provider option is chosen when we jointly have $\frac{a \theta^{2}}{2}-p_{1}<0$ and $\frac{a}{2}\left(\left(\theta-q_{2}\right)^{+}\right)^{2}-p_{2}<0$. Those two inequalities are equivalent to $\theta<\sqrt{\frac{2 p_{1}}{a}}$ and $\theta<q_{2}+\sqrt{\frac{2 p_{2}}{a}}$, respectively, giving $i$ ). We now assume that $\theta \geq \theta^{-}$, i.e., one provider is selected.

- If $\sqrt{\frac{2 p_{1}}{a}} \leq q_{2}+\sqrt{\frac{2 p_{2}}{a}}$, then the volume-based provider could be preferred to the flat-rate if $\frac{a}{2}\left(\left(\theta-q_{2}\right)^{+}\right)^{2}-p_{2}>\frac{a \theta^{2}}{2}-p_{1}$, that is, if $\theta<\frac{p_{1}-p_{2}}{a q_{2}}+\frac{q_{2}}{2}$. However, the condition $\sqrt{\frac{2 p_{1}}{a}} \leq$ $q_{2}+\sqrt{\frac{2 p_{2}}{a}}$ is equivalent to $p_{1} \leq \frac{a}{2} q_{2}^{2}+p_{2}+a q_{2} \sqrt{\frac{2 p_{2}}{a}}$, which yields $\frac{p_{1}-p_{2}}{a q_{2}}+\frac{q_{2}}{2} \leq q_{2}+\sqrt{\frac{2 p_{2}}{a}}$. Hence $\theta \geq \sqrt{\frac{2 p_{1}}{a}}$ implies that $\theta \geq \frac{p_{1}-p_{2}}{a q_{2}}+\frac{q_{2}}{2}$ : the volume-based provider is therefore never chosen here.

- Now assume that $q_{2}+\sqrt{\frac{2 p_{2}}{a}}<\sqrt{\frac{2 p_{1}}{a}}$. For $\theta \geq \theta^{-}$the volume-based provider is selected when $\theta<\frac{p_{1}-p_{2}}{a q_{2}}+\frac{q_{2}}{2}$; above that threshold, the flat-rate is preferred.

Figure 1 summarizes the two situations for the repartition of users.
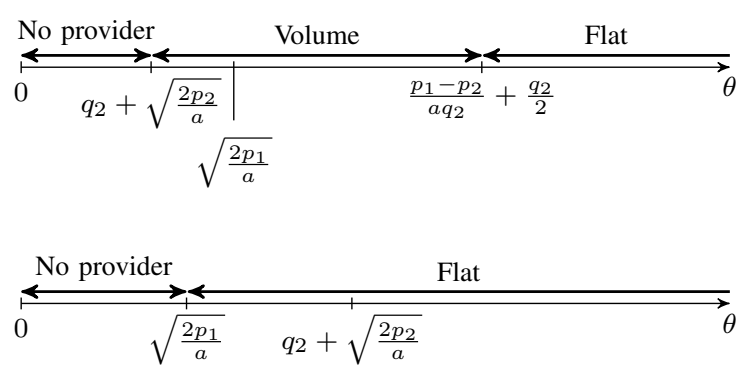

Fig. 1: The two situations for the repartition of users

As an illustration, we draw in Figure 2a the curves of $V\left(\theta, v_{\max }(\theta)\right)-p_{1}$ and $V\left(\theta, v_{2}^{*}(\theta)\right)-p_{2}-q_{2} v_{2}^{*}(\theta)$, i.e., the utilities that type- $\theta$ users would get from choosing Provider 1 or 2 , respectively, for the parameter values $a=2, p_{2}=$ $0, p_{1}=0.002, q_{2}=0.02 . \quad$ In this case, $\sqrt{\frac{2 p_{1}}{a}}=0.0447>$ $q_{2}+\sqrt{\frac{2 p_{2}}{a}}=0.02$, and we indeed observe that under 0.02 , no provider is chosen, the volume-based provider is the best option between 0.02 and $\frac{p_{1}-p_{2}}{a q_{2}}+\frac{q_{2}}{2}=0.06$, and the flat-rate one should be chosen above this last value.

We also draw in Figure $2 \mathrm{~b}$ the case when $q_{2}=0.06$. For those parameter values, $\sqrt{\frac{2 p_{1}}{a}}=0.0447<q_{2}+\sqrt{\frac{2 p_{2}}{a}}=$ 0.06 , and we indeed observe that under 0.0447 , no provider is chosen, and only the flat-rate one above this value.

In both cases, the curve for the volume-based provider starts at 0 because $p_{2}=0$, but any strictly positive value of $p_{2}$ would lead to negative utilities for low $\theta$ values. We however say that no ISP is chosen when the user is indifferent between "no ISP" and "ISP 2" here, because the consumed quantity with ISP 2 would be zero. 


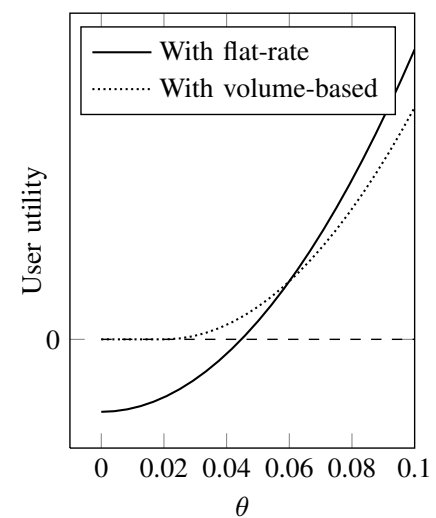

(a) $q_{2}=0.02$

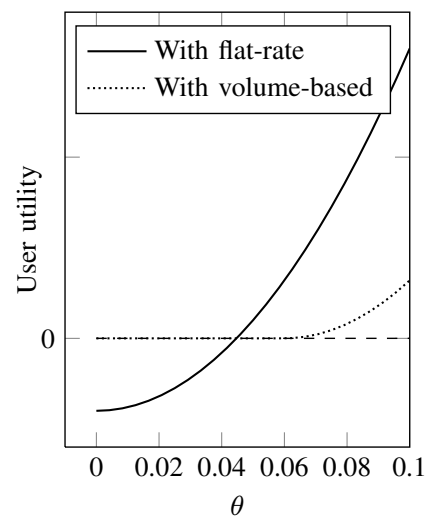

(b) $q_{2}=0.06$
Fig. 2: Utility functions for $a=2, p_{2}=0, p_{1}=0.002$.

\section{DETERMINATION OF PRICES AT THE PROVIDER LEVEL}

We now consider the provider point of view, and investigate the pricing decisions that revenue-driven providers would make. To do so we first express the revenue for each offer depending on the pricing parameters, then examine a numerical example of the expected outcome when both options are proposed by the same entity, and when they come from competing provider who may engage in a price war.

\section{A. Expression of the revenue from the two pricing plans}

Let $m_{F}$ (resp., $m_{V}$ ) be the mass of users associated to the flat-rate (resp., volume-based) charging plan. We have

$$
\begin{aligned}
& m_{V}=\int_{\left[\theta^{-}, \theta^{+}\right)} f(\theta) d \theta=F\left(\theta^{+}\right)-F\left(\theta^{-}\right) \\
& m_{F}=\int_{\left[\theta^{+}, \infty\right)} f(\theta) d \theta=1-F\left(\theta^{+}\right)
\end{aligned}
$$

and the corresponding revenues of providers are

$$
\begin{aligned}
& R_{V}=\int_{\left[\theta^{-}, \theta^{+}\right)}\left(p_{2}+q_{2} v_{2}^{*}(\theta)\right) f(\theta) d \theta \\
& R_{F}=p_{1} m_{F}=p_{1}\left(1-F\left(\theta^{+}\right)\right) .
\end{aligned}
$$

Since analytical expressions for those revenues become very heavy even for simple distributions of $\theta$, we will present a numerical study in the remainder of this section, to highlight some phenomena that can occur.

\section{$B$. When the two pricing schemes come from a single provider}

We assume in the subsection that the two options are proposed by the same provider. Offering more options may lead to higher revenues because it can allow the provider to segment the market by designing offers targeted at different types of users. The goal is then to find prices maximizing the total gain:

$$
\max _{p_{1}, p_{2}, q_{2} \geq 0} R_{F}+R_{V}
$$

We consider two different cases, with $a=2$ in both cases.
1) Log-normal distribution for $\theta$ : In a first case, $\theta$ is lognormally distributed with $\mu=-1$ and $\sigma=0.2$. With that type of distribution, the density has a mode, and for those values, we numerically compute the maximum revenue for the provider:

$$
\max _{p_{1}, p_{2}, q_{2} \geq 0} R_{F}+R_{V}=8.373 \times 10^{-2}
$$

obtained at $p_{1}=0.137, p_{2}=4.43 \times 10^{-2}, q_{2}=9.36 \times 10^{-2}$.

Under those values, we get from Proposition 1 that for $\theta<\theta^{-}=0.304$ users do not subscribe to the service, between $\theta^{-}$and $\theta^{+}=0.542$ they choose the volume-based provider, and the flat-rate one above $\theta^{+}$. This results in masses $m_{V}=$ 0.803 and $m_{F}=0.0264$, and revenues $R_{V}=0.0801$ and $R_{F}=0.00361$. Hence most revenue comes from the volumebased offer, that is also selected by most users, the flat-rate alternative being used by the biggest users.

For comparison purposes, let us look at the provider revenue, had he offered only one option. If only flat rate was proposed, the optimal price would be $p_{1}=0.1012$ leading to a revenue $7.7547 \times 10^{-2}$, while if we only had a volumebased option, the optimal prices would be $p_{2}=4.55 \times 10^{-2}$ and $q_{2}=9.0 \times 10^{-2}$ leading to a revenue $8.3713 \times 10^{-2}$. The gain from proposing the two offers is minor here; we do not claim that it will systematically be the case though. The two offers nevertheless allow big users to consume as wished: those with the flat rate option consume a volume $\int_{\theta^{+}}^{\infty} v_{\max }(\theta) f(\theta) d \theta=\int_{0.54196}^{\infty} a \theta f(\theta) d \theta=0.0309$, which was only $\int_{\theta^{+}}^{\infty} v_{2}^{*}(\theta) f(\theta) d \theta=0.02597$ when there was only the volume-based option. Hence a $19 \%$ increase of aggregated volume for those "high- $\theta$ " users. Remark, to see the proportions, that the total volume for the volume-based option is $\int_{\theta^{-}}^{\theta^{+}} v_{2}^{*}(\theta) f(\theta) d \theta=0.4758$.

2) Exponential distribution for $\theta$ : If on the other hand, $\theta$ is exponentially distributed with rate 1 , then we numerically compute the maximum revenue

$$
\max _{p_{1}, p_{2}, q_{2} \geq 0} R_{F}+R_{V}=7.358 \times 10^{-2}
$$

obtained at $p_{1}=43.35, p_{2}=0, q_{2}=1.0$.

Here, except for a very negligible proportion of the population, only the volume-based scheme is chose. More precisely, $\theta^{-}=1$ and $\theta^{+}=22.175$, which results in masses (proportions) $m_{V}=0.3679$ and $m_{F}=2.34 \times 10^{-10}$, and revenues $R_{V}=0.7358$ and $R_{F}=1.015 \times 10^{-8}$. We also observe here a negative aspect of monopolies, that is demand reduction with respect to competitive situations: the provider sets prices such that less than $37 \%$ of users finally subscribe to one plan.

Again, the gain from offering two schemes instead of one is very small: If we had a flat rate offer only, the optimal price would be $p_{1}=3.9998$, leading to a revenue 0.54134 , while with volume-based only the optimal prices would have been $p_{2}=0$ and $q_{2}=1.0$ leading to the same revenue as in the two-scheme situation.

\section{Pricing game when the two options are offered by providers in competition}

We consider now that the two options are offered by two ISPs in competition, each one trying to maximize its own revenue. We then have a non-cooperative game where both 
providers play with their price (respectively $p_{1}$ and $\left(p_{2}, q_{2}\right)$ for the flat-rate and volume-based providers).

1) Without any cost: price war: Figure 3a displays the best responses of players when the flat-rate provider plays with $p_{1}$ and the volume-based one with $q_{2}$ (taking $p_{2}=0$ ) and $F$ is the cdf of a log-normal distribution with $\mu=-1$ and $\sigma=0.2$. It can be readily seen that there is no intersection point, so that we end up with a price war: players reduce their price to attract customers, leading to zero prices.

The same result is obtained in the case where $\theta$ follows an exponential distribution with rate 1 .

2) Game with management costs: Now, let us consider the case when we subtract a cost $\mathrm{cm}_{V}$ and $\mathrm{cm}_{F}$ to the revenues of respectively the volume-based and flat providers, corresponding to a management cost $c$ per unit of mass of customers.

In Figure 3b, we draw the best-response curves of both ISPs when the flat-rate provider plays with $p_{1}$ and the volumebased one with $q_{2}$ (taking $p_{2}=0$ ) and the $\theta$ parameters are log-normally distributed with $\mu=-1$ and $\sigma=0.2$. In this

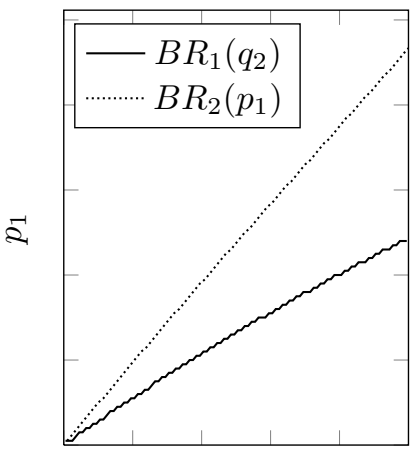

$q_{2}$

(a) No management cost

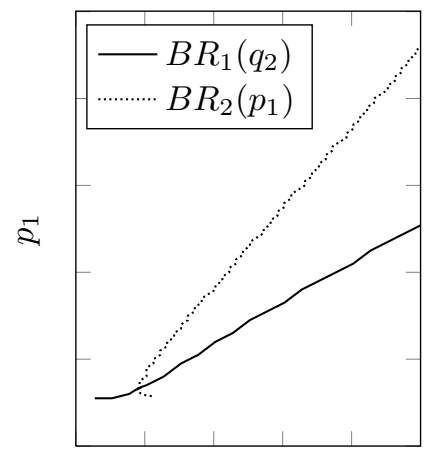

$q_{2}$

(b) Management cost $c=0.01$
Fig. 3: Best-responses when $\theta$ s are log-normally distributed.

figure, we only draw the best responses leading to a positive revenue, hence there is no best-response when the price set by the opponent is too low.

A Nash equilibrium is then obtained at

$$
\left(q_{2}=0.018104, p_{1}=0.01319\right)
$$

giving revenues (taking into account the costs) $R_{F}=$ 0.00150139 and $R_{V}=4.70877 \times 10^{-4}$. Note that these revenues are very small when compared to the monopoly case for which, with this management cost, we obtain an optimal revenue $R_{F}+R_{V}=0.075583$ at $p_{1}=0.143, p_{2}=0.049$ and $q_{2}=0.0895$. In this competition case, $m_{V}=0.5293$ and $m_{F}=0.4707$ : all users subscribe to an offer (because $p_{2}=0$ ), and much more users than in the case of a monopoly choose the flat-rate option.

The decreasing behavior of $B R_{2}\left(p_{1}\right)$ before starting increasing is intriguing. Figure 4 shows the revenue of the volume-based provider in terms of $q_{2}$ for three values of

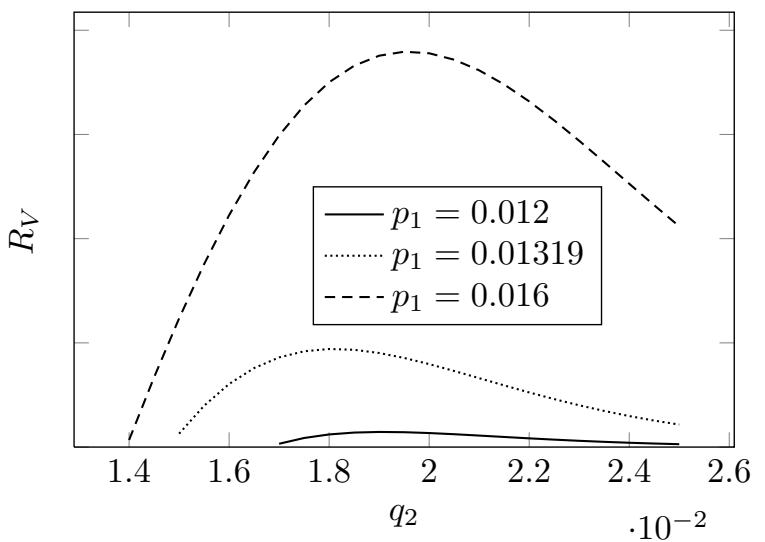

Fig. 4: Revenue for different values of $p_{1}$ when when $\theta$ s are $\log$ normally distributed and with a management cost $c=0.01$.

$p_{1}$, and confirms that the value of $q_{2}$ optimizing $R_{V}$ is not monotonous in $p_{1}$. To further understand what happens, we plot in Figure 5 the thresholds $\theta^{-}$(that is the same for the three values of $p_{1}$ selected) and $\theta^{+}$, recalling that users with $\theta \in\left[\theta^{-}, \theta^{+}\right)$select ISP 2 . An explanation for this phenomenon comes from the fact that $q_{2}$ affects both $\theta^{-}$(positively) and $\theta^{+}$(negatively). Among the users with parameter close to $\theta^{-}$, there may be some "costly clients" with small consumption, whose impact on cost exceeds the additional revenue, but as illustrated in Figure 5, ISP 2 cannot deter them from entering the system (by increasing $\theta^{-}$) without losing "money-making" customers to the competitor (by reducing $\theta^{+}$). But when $p_{1}$ decreases, $\theta^{+}$also decreases and the the savings on deterring costly clients from subscribing by increasing $q_{2}$ may now exceed the revenue from money-making customers that ISP 2 would lose: in that case ISP 2 is better off increasing $q_{2}$. This is possible here even if the slope of $\theta^{-}$is very small, because the log-normal distribution has a mode, hence a small change in $\theta^{-}$may affect a significant proportion of users (the "costly" ones). This phenomenon justifies the introduction of a non-null fixed part $p_{2}$ in the volume-based pricing scheme, as a way for ISP 2 to avoid those costly clients.

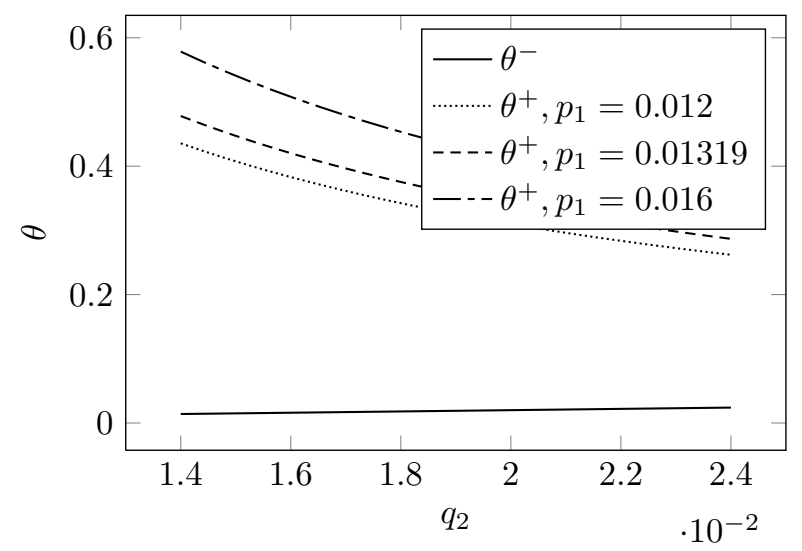

Fig. 5: Threshold values when $\theta$ s are log normally distributed and with a management cost $c=0.01$. 


\section{CONCLUSIONS AND FUTURE WORK}

This paper investigates the coexistence of flat-rate and volume-based pricing, where the choice is left to users, but the pricing parameter values are set by revenue-maximizing providers. When both options are controlled by a monopolist provider, our results indicate that the possibility of user segmentation offered by the variety of schemes does not bring large revenue improvements to the provider. On the other hand, if each option is offered by an independent provider, competition drives prices down, questioning the survivability of the providers.

An aspect worth including in future models is the preference of users for flat-rate schemes when the expected price difference with volume-based is low. That preference, apparently contradicting the user rationality assumed in this paper, can be explained by users' aversion to risk and to having to monitor one's consumption. Other directions to investigate include the study of other types of schemes, such as the one proposed in [7] where a flat rate is applied but users can additionally purchase higher-quality service, charged on volume.

\section{REFERENCES}

[1] A. Odlyzko, B. St. Arnaud, E. Stallman, and M. Weinberg, "Know your limits: Considering the role of data caps and usage based billing in internet access service,” Public Knowledge, Tech. Rep., May 2012.

[2] P. Maillé and B. Tuffin, Telecommunication Network Economics: From Theory to Applications. Cambridge University Press, 2014.

[3] D. Songhurst, Ed., Charging Communication Networks. Elsevier, 1999.

[4] J. Mo, W. Kim, and D. Lee, "Impacts of universal service regulation for broadband internet services," in Economics of Converged, Internet-Based Networks, ser. Lecture Notes in Computer Science, J. Cohen, P. Maillé, and B. Stiller, Eds. Springer Berlin Heidelberg, 2011, vol. 6995, pp. 14-25

[5] M. Cho and M. Choi, "Pricing for mobile data services considering service evolution and change of user heterogeneity," IEICE Transactions on Communications, vol. E96-B, no. 2, pp. 543-552, 2013.

[6] - "Pricing mobile data services of different quality levels," in Proc. of 3rd International Conference on ICT Convergence (ICTC), Jeju, South Korea, 2012.

[7] J. Altmann and K. Chu, "How to charge for network services-flat-rate or usage-based?" Computer Networks, vol. 36, no. 5, pp. 519-531, 2001. 